\title{
Enhancing Immediate Memory, Potential Learning, and Working Memory with Transcranial Direct Current Stimulation in Healthy Older Adults
}

\author{
Encarnacion Satorres ( $\nabla$ encarna.satorres@uv.es ) \\ University of Valencia \\ Juan Carlos Melendez \\ University of Valencia \\ Alfonso Pitarque \\ University of Valencia \\ Elena Real \\ University of Valencia \\ Mireia Abella \\ University of Valencia \\ Joaquin Escudero \\ Hospital General Universitario De Valencia
}

\section{Research Article}

Keywords: tDCS, healthy older adults, immediate memory, working memory, learning potential.

Posted Date: February 3rd, 2022

DOI: https://doi.org/10.21203/rs.3.rs-1163460/v1

License: (1) This work is licensed under a Creative Commons Attribution 4.0 International License.

Read Full License 


\section{Abstract \\ Background}

Transcranial direct current stimulation (tDCS) has emerged as a prevention method or minimizer of the normal cognitive deterioration that occurs during the aging process. tDCS can be used to enhance cognitive functions such as immediate memory, learning, or working memory in healthy subjects.

\section{Objective}

The purpose of this study was to analyze the effect of two 20-minute sessions of anodal transcranial direct stimulation on immediate memory, learning potential, and working memory in healthy older adults.

\section{Methods}

A randomized, single-blind, repeated-measures, placebo-controlled design was used. The sample is made up of 31 healthy older adults, of whom 16 were in the stimulation group and 15 in the sham group The anode was placed on position F7, coinciding with the left dorsolateral prefrontal cortex region, and the cathode was placed on Fp2, the right supraorbital area (rSO).

\section{Results}

When comparing the results of the treatment group and the sham group, differences were observed in working memory and learning potential; however, no differences in immediate memory were found.

\section{Conclusion}

The results showed that tDCS is a non-invasive and safe tool to enhance cognitive processes in healthy older adults interested in maintaining some cognitive function.

\section{Introduction}

Cognitive skills play an important role in the daily functioning of older adults. However, some of these cognitive abilities decline during the aging process. In normal aging, one of the main signs of deterioration is memory loss, which affects both immediate and long-term memory [1]. In aging, there is also a decrease in executive functions (EF), that is, higher-level cognitive skills such as planning, problem solving, and working memory (WM), all of which are necessary to perform daily activities and maintain independence with age [2]. In fact, working memory is related to other frontally controlled cognitive 
functions such as language or learning, and so stimulating these cognitive functions will produce more complex actions and thoughts [3].

Recently, transcranial direct current stimulation (tDCS) has emerged as a prevention method or minimizer of the normal cognitive deterioration that occurs during the aging process. tDCS is a non-invasive brain stimulation technique that is increasingly being used to modulate neuronal activity [4]. The action mechanism of tDCS is based on the principles of neuronal plasticity, given that it is induced through the generation of a sub-threshold stimulation polarity-dependent alteration of membrane potentials, modifying spontaneous discharge rates. Cortical excitability is modulated, resulting in either hypopolarization/excitation or hyperpolarization/inhibition, depending on the polarity of the stimulation [5].

A number of studies have demonstrated that tDCS can be used to enhance cognitive functions in healthy subjects, and it has been employed to improve memory, learning, or working memory. These studies have mainly applied stimulation on the left dorsolateral prefrontal cortex (DLPFC) to improve cognitive performance, and they have shown its effectiveness in both healthy subjects and neuropsychiatric samples such as patients with Mild Cognitive Impairment or Alzheimer's disease [6]. However, other studies have also found more modest or contradictory results [7]. Inconsistent findings might result from methodological differences or low statistical power. In addition, this diversity in the results could be explained by the large variability in stimulation parameters, such as the number of electrodes and their position, current intensity, session duration, and the use of "offline" versus "online" protocols [8].

Prehn et al. [9] found that a combination of selective serotonin reuptake inhibitors (SSRI) and tDCS could produce significant improvements in immediate memory in older participants, whereas Bystad et al. [10] observed no significant differences between placebo and active tDCS on verbal memory functions. It is unclear whether tDCS actually leads to cognitive changes in immediate memory in healthy older adults, and the effects have been associated with conflicting results. Hsu et al. [11] argue that tDCS should offer more satisfactory results in populations with pathologies because there may be a ceiling effect in healthy participants. However, some studies confirm the efficacy of anodic tDCS, noting that these effects are due to improved recovery [12] or even consolidation [13]. Sandrini et al. [14]. point out that better recall performance might be explained by more efficient coding, but this is an open question.

The ability to learn and remember new information declines with age [15]. A study using tDCS showed that anodal tDCS can facilitate learning as well as word generation [16]. Javadi and Walsh showed that application of anodal tDCS over the left DLPFC during the encoding phase can enhance performance on verbal memorization [17]. For declarative verbal learning and memory specifically, research has focused on stimulating the DLPFC. Nikoli et al. [18]. found that focal anodal tDCS stimulation of the DLPFC increased the rate of verbal learning compared to sham. These authors suggest that an increase in working memory efficiency may therefore account for the significantly faster rate of learning observed on declarative verbal memory tasks. 
However, results for working memory performance are mixed. This is not surprising due to the small samples, the wide range of working memory tasks, and the different stimulation protocols used. In healthy participants, repeated sessions of anodal tDCS over the DLPFC [19] or a single session $[20,18]$ enhanced verbal working memory. Nikolin et al. (2018) suggest that tDCS does not produce substantial improvements in working memory performance in healthy participants [21]. In addition, the information provided by meta-analyses is contradictory. Hill et al. [22]. found only partial support for the hypothesis of an enhancement effect of anodal tDCS on working memory performance, noting that reaction times were significantly improved on offline WM tasks. Dedoncker et al. found that healthy participants responded significantly faster [8]. However, when pooling studies that evaluated the effects of a single tDCS session, Galli et al.'s results add to a growing body of meta-analytical work that failed to show an effect of tDCS on working memory accuracy [23].

The aim of the present study was to investigate the effects of tDCS on verbal memory. Based on previous research, and due to the contradictory findings on immediate memory, learning, and working memory, we aimed to analyse the effect of two sessions of anodal stimulation on these variables, and the left DLPFC was selected as the main stimulation site. Therefore, we hypothesize that the application of tDCS will have beneficial effects on verbal cognition in older adults, producing a significant increase in the scores of the stimulation group compared to the placebo group.

\section{Material And Methods}

\section{Participants}

Recruitment was carried out in a university program for seniors (NAU Gran) at the University of Valencia. To be eligible for inclusion, participants had to be over 65 years of age, have no cognitive impairment, and be able to attend two sessions on consecutive days. Initially, 33 healthy older adults were recruited, but two were dropped for not attending consecutive sessions. The sample was composed of 31 healthy older adults ( 16 women, 15 men) between 65 and 80 years old $(M=69.9, S D=4.1$ ) who participated voluntarily and signed the informed consent before starting the study. The Ethical Committee on Human Research of the University of Valencia approved this study.

A randomized, single-blind, repeated-measures, placebo-controlled design was used. Subjects were randomly assigned to one of two groups where they received either tDCS or sham stimulation. The stimulation group was made up of 16 older adults ( 8 women, 8 men) between 65 and 78 years old ( $M=$ $69.8, S D=3.4$ ); the sham group consisted of 15 older adults ( 8 women, 7 men) between 65 and 80 years old $(M=70.13, S D=4.7)$. The groups were equal in their sociodemographic characteristics, with no differences in age $(t(29)=.217, p=.830)$, gender $\left(\chi^{2}=0.027, p=.987\right)$, or academic level (U of MannWhitney $=108, p=.607$ ).

\section{Instruments}


The score on the Mini-Mental State Examination [24] was used as an inclusion criterion to rule out participants with cognitive impairment. None of the participants showed cognitive impairment, and the mean for the entire group was $29.8(S D=.54$, range 28-30). Furthermore, no significant differences were observed at baseline between the stimulation and sham groups $(t(29)=.592, p=.559)$.

The Complutense Verbal Learning Test (TAVEC) [25] consists of a list of 16 words that are presented to the subject five times in order to evaluate different memory and learning processes. Each trial is scored from 0 to 16 , with a maximum total score of 80 . The score on the first trial provides a measure of immediate memory or short-term free memory, and the fifth trial assesses the learning of the word list after training it five consecutive times. In addition, learning potential was evaluated according to Kliegl et al. [26]. This score is obtained by calculating the difference in the words remembered between the first and fifth trials. An increase in the score indicates a higher level of cognitive plasticity.

Digits forward and digits backward from the Wechsler Intelligence Scale for Adults-III (WAIS-III) [27]. The digits forward task assesses immediate recall. It requires the participant to repeat a sequence of numbers in the same order in which they were read. On the digits backward task, the subject must repeat a sequence of numbers in the reverse order of their presentation. The task is used to assess working memory. Both subtests have eight elements with two items each. The test ends when the subject fails on two items in the same element. For each correct item, one point is given, with a maximum score of 16 on each subtest.

Because the assessment of participants was carried out on two consecutive days, it was necessary to select different versions of these two tests to eliminate any bias due to learning.

\section{Procedure}

All participants were contacted through the teaching program carried out at the University of Valencia for older adults. The objective of the study was explained to them, and their voluntary participation was requested, after informing them that they would have to attend two consecutive sessions. Once the list of participants had been obtained, an adapted appointment calendar was established, and they were randomly assigned to the stimulation or sham groups.

HDC stimulator (Newronika TM, Milan, Italy) was used to perform non-invasive tDCS with a constant current intensity of $2 \mathrm{~mA}$. Two electrodes with sponges soaked in saline solution $(5 \times 5 \mathrm{~cm})$ were used. The anode was placed on position F7, coinciding with the left dorsolateral prefrontal cortex region, and the cathode was placed on Fp2, coinciding with the right supraorbital area (rSO). The stimulation time was 20 minutes, with an initial and final ramp of 30 seconds so that the participant could adapt to the sensation of the current. The sham or placebo group received direct current only on the ramps to generate a sensation of the effect.

The first session began by reviewing the objectives of the study and completing the informed consent. Next, the evaluation protocol was administered. After that, the first session of tDCS stimulation began. 
The second session began with the application of tDCS stimulation, and after approximately three minutes of stimulation, the evaluation protocol was administered.

\section{Analysis}

For the comparison of the groups, $t$ tests and Mann-Whitney tests for independent samples were used. For the comparison of the baseline and post-treatment measurements of the two groups, mixed ANOVAS with 2 sessions (before versus after treatment; within subjects) X 2 groups (treatment versus control; between subjects) were performed. The data were analyzed with SPSS 21.

\section{Results}

A mixed ANOVA with 2 sessions (Before vs After treatment; within subjects) X 2 groups (Treatment vs Control; between subjects) was performed on the scores of the TAVEC first trial, which assesses immediate memory, obtaining a significant main effect of the sessions $\left(F(1,29)=4.87, p<.035, \eta^{2} p=\right.$ $0.144)$, but not a main effect of the group $\left(F(1,29)=1.63, p=.212, \eta^{2} p=0.144\right)$ or the session $\mathrm{X}$ group interaction $\left(F(1,29)=.005, p=.944, \eta^{2} p=0.001\right.$; Treatment: $M_{\text {PRETEST }}=5.37$, $M_{\text {POSTETS }}=5.81$; Control: $\left.M_{\text {PRETEST }}=4.66, M_{\text {POSTETS }}=5.13\right)$.

Regarding the fifth trial of the TAVEC, a mixed ANOVA with 2 sessions $X 2$ groups was also performed, showing that the main effect of the sessions variable was significant $\left(F(1,29)=45.82, p<.001, \eta^{2} p=\right.$ $0.612)$, as well as the session $X$ group interaction $\left(F(1,29)=26.79, p<.001, \eta^{2} p=0.480\right)$, but not the main effect of the group $\left(F(1,29)=1.34, p=.256, \eta^{2} p=0.044\right)$. A simple effects test conducted to analyse the significant session $X$ group interaction showed that the means of the treatment and control groups did not significantly differ on the pre-test $\left(F(1,29)=.012, p=.915, \eta^{2} p=0.001 ; M_{\text {TREATMENT }}=11.5\right.$ vs $\left.M_{\text {CONTROL }}=11.6\right)$, but the treatment group showed a significantly higher mean on the post-test $(F(1$, $29)=6.44, p=.017, \eta^{2} p=0,182 ; M_{\text {TREATMENT }}=14$ vs $\left.M_{\text {CONTROL }}=11.93\right)$. A comparison of the pre-test and post-test scores showed that, in the control group, there was no significant change $(F(1,29)=1.22, p=$ $\left..277, \eta^{2} p=0.041\right)$ in the means $\left(M_{\text {PRETEST }}=11.6\right.$ vs $\left.M_{\text {POSTETS }}=11.93\right)$, whereas in the treatment group, a significant increase $\left(F(1,29)=73.72, p<0,001, \eta^{2} p=0.718\right)$ in the means $\left(M_{\text {PRETEST }}=11.5\right.$ vs $M_{\text {POSTETS }}=$ 14) was observed after the application of the stimulation.

In relation to the total words learned on the TAVEC after the five trials, a mixed ANOVA was also performed, obtaining a significant main effect of the sessions $\left(F(1,29)=33.62, p<.001, \eta^{2} p=0.537\right)$ and the session $X$ group interaction $\left(F(1,29)=26.64, p<.001, \eta^{2} p=0.479\right)$, but the main effect of the group was not significant $\left(F(1,29)=1.64, p=.210, \eta^{2} p=0.054\right)$. A simple effects test performed to analyse the significant interaction showed that there was no difference between the groups on the pretest $\left(F(1,29)=.21, p=.654, \eta^{2} p=0.007 ; M_{\text {TREATMENT }}=45.18\right.$ vs $\left.M_{\text {CONTROL }}=43.33\right)$, but on the post-test the difference between the groups was significant $\left(F(1,29)=4.45, p=.044, \eta^{2} p=0.133 ; M_{\text {TREATMENT }}=\right.$ 52.06 vs $\left.M_{\text {CONTROL }}=43.73\right)$, due to the increase in the scores of the treatment group. Comparison of the 
pre-test and post-test scores did not show a significant difference in the control group $(F(1,29)=.19, p=$ $.660, \eta^{2} p=0.007 ; M_{\text {PRETEST }}=43.33$ vs $\left.M_{\text {POSTETS }}=43.73\right)$. However in the treatment group, a significant increase $\left(F(1,29)=62.06, p<.001, \eta^{2} p=0.682 ; M_{\text {PRETEST }}=45.18\right.$ vs $\left.M_{\text {POSTETS }}=52.06\right)$ was observed in the means for the total number of words after stimulation.

Learning potential was calculated as the difference between trials 5 and 1 on the TAVEC, and mixed ANOVAs were performed for its analysis, obtaining a significant main effect of the session $(F(1,29)=$ $\left.4.43, p=.044, \eta^{2} p=0.133\right)$ and the session $X$ group interaction $\left(F(1,29)=15.96, p<.001, \eta^{2} p=0.355\right)$, but the group effect was not significant $\left(F(1,29)=.03, p=.847, \eta^{2} p=0,001\right)$. The simple effects tests carried out to analyze the significant interaction showed that there were no significant differences between the groups on the pre-test $\left(F(1,29)=2.51, p=.124, \eta^{2} p=0.079 ; M_{\text {TREATMENT }}=6.25\right.$ vs $M_{\text {CONTROL }}$ $=7.4)$. However, significant differences were observed on the post-test $\left(F(1,29)=4.55, p=.041, \eta^{2} p=\right.$ $0.136 ; M_{\text {TREATMENT }}=8.18 \mathrm{vs} M_{\text {CONTROL }}=6.8$ ), due to the increase in the mean of the treatment group. Comparison of the pre-test and post-test scores in each group revealed that, in the control group, there was no significant change in their scores $\left(F(1,29)=1.73, p=.199, \eta^{2} p=0.056 ; M_{\text {PRETEST }}=7.4\right.$ vs $M_{\text {POSTETS }}=6.8$ ), whereas the treatment group showed a significant increase from pre-test to post-test $\left(F(1,29)=19.24, p<0,001, \eta^{2} p=0.399 ; M_{\text {PRETEST }}=6.25\right.$ vs $\left.M_{\text {POSTETS }}=8.2\right)$ after stimulation application.

Regarding the direct digits, mixed ANOVAs were performed, obtaining a significant main effect of the session $\left(F(1,29)=6.32, p=.018, \eta^{2} p=0.179\right)$, but not the group $\left(F(1,29)=.12, p=.126, \eta^{2} p=0,004\right)$ or the session X group interaction $\left(F(1,29)=2.12, p=.156, \eta^{2} p=0.068\right.$; Treatment: $M_{\text {PRETEST }}=10.06$, $M_{\text {POSTETS }}=11.06$; Control: $\left.M_{\text {PRETEST }}=10.13, M_{\text {POSTETS }}=10.4\right)$.

Finally, the mixed ANOVA of the inverse digits showed a significant main effect of the session $(F(1,29)=$ 9.57, $\left.p=.004, \eta^{2} p=0.248\right)$ and the session $\mathrm{X}$ group interaction $\left(F(1,29)=5.33, p=0,028, \eta^{2} p=0.155\right)$, but the main group effect was not significant $\left(F(1,27)<.01, p=.964, \eta^{2} p=0.001\right)$. The simple effects tests performed to analyze the significant interaction showed that there were no significant differences between the groups on the pre-test $\left(F(1,29)=.43, p=.513, \eta^{2} p=0.015 ; M_{\text {TREATMENT }}=7.31\right.$ vs $M_{\text {CONTROL }}=$ $7.86)$ or on the post-test $\left(F(1,29)=.75, p=.395, \eta^{2} p=0.025 ; M_{\text {TREATMENT }}=8.68\right.$ vs $\left.M_{\text {CONTROL }}=8.06\right)$. The study of the change in each group revealed that, whereas in the control group there was no significant change in the scores between the sessions $\left(F(1,29)=.29, p=.589, \eta^{2} p=0.010\right)\left(M_{\text {PRETEST }}=7.86 \mathrm{vs}\right.$ $\left.M_{\text {POSTETS }}=8.06\right)$, the treatment group showed a significant change $\left(F(1,29)=15.08, p=.001, \eta^{2} p=\right.$ 0.342 ) after the application of the stimulation, with an increase in their scores ( $M_{\text {PRETEST }}=7.31 \mathrm{vs}$ $M_{\text {POSTETS }}=8.68$ ).

\section{Discussion}

The present study aimed to demonstrate the effectiveness of the application of tDCS in healthy older adults. When comparing the results of the treatment group and the sham group, differences in working 
memory and learning potential were found, but no differences in immediate memory were observed.

For immediate memory, the results of the current study were not expected, given that no statistically significant differences were observed between the treatment group and the sham group. A possible justification for this lack of evidence is provided by Tonegawa et al. (2015), who argue that there is no particular structure specialized in the function of memory because it is actually stored in patterns of connectivity in different areas [28]. In addition, various regions, including the DLPFC, are coordinated in the processing, encoding, and retrieval of verbal information $[29,30]$. In this way, targeting a single structure can be a limitation of the study [28].

Furthermore, to assess immediate memory, the task used in the present study was the recall of forward digits and free recall words.

On the recall of words, one of the possible justifications is the influence of the familiarity factor, which is considered a source of false memories and increases with age [31]. Scalici et al. point out that recollection and familiarity depend, among other areas, on the prefrontal cortex [32]. Specifically, Aly et al. and Scalici et al. observed that the left DLPFC is specialized in the familiarity factor, the area stimulated in the present study $[33,32]$. Therefore, it is logical that the expected results were not obtained for immediate memory, given that the area specialized in familiarity, and not in memory, was stimulated.

Regarding the learning potential, the hypothesis that the group that received tDCS would have a higher rate of correctly remembered items compared to the sham group was confirmed. Perceval et al. studied verbal associative learning and its long-term effects in healthy older adults [34]. Improvements in learning and memory capacity were found, but only in the group of older adults whose initial learning performance was lower. Thus, these results show that the short-term and long-term effects of tDCS depend on the baseline cognitive state and particularly benefit older adults who may need help.

Neuroplasticity is based on cortical and neuronal excitability, and stimulating excitability with tDCS enhances cognitive processes [35], through the fortification of neural connections [36, 37] and, therefore, a reconfiguration of brain networks [38]. For example, Huo et al. (2020) found that tDCS was effective when stimulating the DLPFC because neural changes in the prefrontal cortex resulted in better memory performance [39].

Regarding working memory, our results are consistent with other studies showing improvements in WM performance with the tDCS technique $[40,41,42,43,44]$, which means that tDCS can help to improve this cognitive ability. Effects of tDCS combined with working memory training have been shown to extend and increase training gains $[42,19,2]$. Thus, $\operatorname{tDCS}$ seems to be a useful intervention technique to reverse the typical working memory decline observed in aging [43].

However, more recent meta-analyses have concluded that the effects are small [22] or partial [7]. Thus, more empirical studies are needed on the impact of tDCS on functional connectivity of the working 
memory network, in order to harness and optimize tDCS as a treatment approach for cognitive decline in older adults [43].

Although there is still no consensus about the reason for the efficacy of tDCS for working memory, the most widely accepted explanation today is that anodal stimulation of the left DLPFC may increase the effectiveness of WM training and be useful when applied before or during WM tests [45]. Moreover, tDCS can improve WM because it modulates excitability and cerebral cortical activity by transmitting a weak electrical current in the brain $[40,7,19]$. A more recent study explained that tDCS selectively modulates frontal functional connectivity of the working memory in older adults [43].

\section{Limitations and future lines}

Some limitations of this study must be acknowledged. First, the intervention consists of only two sessions, which may not be sufficient. Second, cognitive functions were evaluated before and after the intervention to determine whether it had significant effects, but there was no follow-up to find out whether the effects are sustained over time.

As future lines of research, we propose applying this same intervention in different populations. Because its efficacy has been shown in a healthy population for various cognitive functions, it is plausible to imagine that it could also work in cases of incipient cognitive deterioration. Thus, it could serve as an early intervention or when the disease has already manifested itself and achieve an improvement in the quality of life. An intervention protocol that includes a larger number of sessions could also be designed. Furthermore, to find out whether the effects are sustained in the long term, future studies should carry out a follow-up evaluation sometime after the intervention.

\section{Conclusion}

In sum, the efficacy of tDCS was observed for working memory and potential learning variables, whereas for immediate memory no significant differences were found after the intervention. Thus, the results presented show that tDCS is a non-invasive and safe tool to enhance cognitive processes in healthy older adults interested in maintaining cognitive function. However, because the results in the literature are diverse, more evidence is needed about its effectiveness in relation to the stimulated brain areas, in order to reach a consensus about why stimulating these specific areas improves some cognitive processes and not others.

\section{Declarations}

Ethics approval and consent to participate

The study was conducted according to the guidelines of the Declaration of Helsinki and approved by the Institutional Review Board (or Ethics Committee) of University of Valencia (H1526539449220; 7 June 2018). The sample participated voluntarily and signed the informed consent before starting the study. 
Consent for publication

The authors consent to the publication of this manuscript.

Avaiability of data and materials

The datasets generated and/or analyzed during the current study are not publicly available due privacy reasons, but are available from the corresponding author on reasonable request.

Competing interests

The authors declare that they have no conflicts of interest.

Funding

This work was supported by MCIN/AEI /10.13039/501100011033 [Grant PID2019-103956RB-I00] and Conselleria de Innovación, Universidades, Ciencia y Sociedad Digital of Generalitat Valenciana (Spain) [Grant GV/2021/174]

Authors' contributions

E.R. and MA have been mainly in charge of reading updated bibliography on the subject and writing the introduction to the manuscript. A.P. and J.C.M. have been in charge of carrying out the statistical analyzes and writing the results. E.S., M.A. and J.C.M. have contrasted the results obtained with other studies and have written the discussion. J.E. has participated in the selection of the sample and in the design of the stimulation based on tDCS. All the authors carefully read the manuscript and fully approved of it.

Acknowledgement

Not applicable

\section{References}

[1] Summers JJ, Kang N, Cauraugh JH. Does transcranial direct current stimulation enhance cognitive and motor functions in the ageing brain? A systematic review and meta-analysis. Ageing Res Rev 2016;25:42-54. https://doi.org/10.1016/j.arr.2015.11.004

[2] Stephens JA, Berryhill ME. Older adults improve on everyday tasks after working memory training and neurostimulation. Brain Stimul 2016;9:553-59. https://doi.org/10.1016/j.brs.2016.04.001

[3] Jaušovec N, Jaušovec K. (2012). Working memory training: Improving intelligence-changing brain activity. Brain Cogn 2012;79:96-106. https://doi.org/10.1016/j.bandc.2012.02.007 
[4] Fontenau C, Mondino M, Arns M, Baeken C, Bikson M, Brunoni AR, Burke M, Neuvonen T, Padberg F, Pascual-Leone A, Polet E, Ruffini G, Santarnecchi E, Sauvaget A, Schellhorn K, Suaud-Chagny MF, Palm U, Brunelin J. Sham tDCS: A hidden soucre of variability? Reflections for further blinded, controlled trails. Brain Stimul 2019;12:668-73. https://doi.org/10.1016/j.brs.2018.12.977

[5] Westwood SJ, Olson A, Miall RC, Nappo R, Romani C. Limits to tDCS effects in language: Failures to modulate word production in healthy participants with frontal or temporal tDCS. Cortex 2017;86:64-82. https://doi.org/10.1016/j.cortex.2016.10.016

[6] Shin YI, Foerster Á, Nitsche MA. Reprint of: transcranial direct current stimulation (tDCS)- Application in neuropsychology. Neuropsychologia 2015;74: 74-95.

[7] Brunoni AR, Vanderhasselt MA. Working memory improvement with noninvasive brain stimulation of the dorsolateral prefrontal cortex: a systematic review and meta-analysis. Brain Cogn 2014;86:1-9. https://doi.org/10.1016/j.bandc.2014.01.008.

[8] Dedoncker J, Brunoni AR, Baeken C, Vanderhasselt MA. A systematic review and meta-analysis of the effects of transcranial direct current stimulation (tDCS) over the dorsolateral prefrontal cortex in healthy and neuropsychiatric samples: Influence of stimulation parameters. Brain Stimul 2016;9:501-17. https://doi.org/10.1016/j.brs.2016.04.006

[9] Prehn K, Stengl H, Grittner U, Kosiolek R, Ölschläger A, Weidemann A, Flöel A. (2017). Effects of anodal transcranial direct current stimulation and serotonergic enhancement on memory performance in young and older adults. Neuropsychopharmacol 2017;42:551-61. https://doi.org/10.1038/npp.2016.170

[10] Bystad M, Storø B, Gundersen N, Wiik IL, Nordvang L, Grønli O, Rasmussen ID, Aslaksen PM. Can accelerated transcranial direct current stimulation improve memory functions? An experimental, placebocontrolled study. Heliyon 2010;6:e05132. https://doi.org/10.1016/j.heliyon.2020.e05132

[11] Hsu WY, Ku Y, Zanto TP, Gazzaley A. Effects of noninvasive brain stimulation on cognitive function in healthy aging and Alzheimer's disease: A systematic review and meta-analysis. Neurobiol Aging 2015;36:2348-59. https://doi.org/10.1016/j.neurobiolaging.2015.04.016

[12] Manenti R, Brambilla M, Petesi M, Ferrari C, Cotelli M. Enhancing verbal episodic memory in older and young subjects after non-invasive brain stimulation. Front Aging Neurosci 2013;5:49.

https://doi.org/10.3389/fnagi.2013.00049

[13] Flöel A, Suttorp W, Kohl O, Kürten J, Lohmann H, Breitenstein C, Knecht S. Non-invasive brain stimulation improves object-location learning in the elderly. Neurobiol Aging 2012;33:1682-9. https://doi.org/10.1016/j.neurobiolaging.2011.05.007

[14] Sandrini M, Manenti R, Brambilla M, Cobelli C, Cohen LG, Cotelli M. Older adults get episodic memory boosting from noninvasive stimulation of prefrontal cortex during learning. Neurobiol Aging 
2016;39:210-16. https://doi.org/10.1016/j.neurobiolaging.2015.12.010

[15] Floel A, Cohen LG. Contribution of noninvasive cortical stimulation to the study of memory functions. Brain Res Rev 2007;53:250-9. https://doi.org/10.1016/j.brainresrev.2006.08.006

[16] Kincses TZ, Antal A, Nitsche MA, Bártfai O, Paulus W. Facilitation of probabilistic classification learning by transcranial direct current stimulation of the prefrontal cortex in the human.

Neuropsychologia 2004;42:113-17. https://doi.org/10.1016/S0028-3932(03)00124-6

[17] Javadi AH, Walsh V. Transcranial direct current stimulation (tDCS) of the left dorsolateral prefrontal cortex modulates declarative memory. Brain Stimul 2012;5:231-41. https://doi.org/10.1016/j.brs.2011.06.007

[18] Nikolin S, Loo CK, Bai S, Dokos S, Martin DM. Focalised stimulation using high definition transcranial direct current stimulation (HD-tDCS) to investigate declarative verbal learning and memory functioning. Neuroimage 2015;117:11-19. https://doi.org/10.1016/j.neuroimage.2015.05.019

[19] Richmond LL, Wolk D, Chein J, Olson IR. Transcranial direct current stimulation enhances verbal working memory training performance over time and near transfer outcomes. Journal of cognitive neuroscience 2014;26:2443-54. https://doi.org/10.1162/jocn_a_00657

[20] Fregni F, Boggio PS, Nitsche M, Bermpohl F, Antal A, Feredoes E, Marcolin MA, Rigonatti S, Silva MTA, Paulu SW, Pascual-Leone A. Anodal transcranial direct current stimulation of prefrontal cortex enhances working memory. Exp Brain Res 2005;166:23-30. https://doi.org/10.1007/s00221-005-2334-6

[21] Nikolin S, Martin D, Loo CK, Boonstra TW. Effects of tDCS dosage on working memory in healthy participants. Brain Stimul 2018;11:518-27.

[22] Hill AT, Fitzgerald PB, Hoy KE. Effects of anodal transcranial direct current stimulation on working memory: A systematic review and meta-analysis of findings from healthy and neuropsychiatric populations. Brain Stimul 2016;9: 197-208. https://doi.org/10.1016/j.brs.2015.10.006

[23] Galli G, Vadillo MA, Sirota M, Feurra M, Medvedeva A. A systematic review and meta-analysis of the effects of transcranial direct current stimulation (tDCS) on episodic memory. Brain Stimul 2019;12:231241. https://doi.org/10.1016/j.brs.2018.11.008

[24] Folstein M, Folstein S, Mc Hugh P. Mini Mental State. A practical method for grading the cognitive state of patients for the clinical. J Psychiatry Res 1975;12:189-198.

[25] Benedet MJ, Alejandre MÁ. TAVEC: Test de aprendizaje verbal España-Complutense. Madrid: TEA ediciones; 1998

[26] Kliegl R, Smith J, Baltes PB. (1989). Testing-the-limits and the study of adult age differences in cognitive plasticity of a mnemonic skill. Dev Psychol 1989;25:247-56. https://doi.org/10.1037/0012- 
[27] Wechsler D. Wechsler intelligence scale for adults-III (WAIS-III). Madrid: TEA ediciones; 2001

[28] Tonegawa S, Pignatelli M, Roy DS, Ryan TJ. Memory engram storage and retrieval. Curr Res Neurobiol 2015;35:101-09. https://doi.org/10.1016/j.conb.2015.07.009

[29] Borst JP, Anderson JR. Using model-based functional MRI to locate working memory updates and declarative memory retrievals in the fronto-parietal network. Proc Natl Acad Sci U S A 2013;110:1628-33. https://doi.org/10.1073/pnas.1221572110

[30] Pisoni A, Turi Z, Raithel A, Ambrus GG, Alekseichuk I, Schacht A, Paulus W, Antal A. Separating recognition processes of declarative memory via anodal tDCS: Boosting old item recognition by temporal and new item detection by parietal stimulation. PloS one 2015;10:e0123085.

https://doi.org/10.1371/journal.pone.0123085

[31] Devitt AL, Schacter DL. False memories with age: Neural and cognitive underpinnings. Neuropsychologia 2016;91:346-59. https://doi.org/10.1016/j.neuropsychologia.2016.08.030

[32] Scalici F, Caltagirone C, Carlesimo GA. The contribution of different prefrontal cortex regions to recollection and familiarity: A review of fMRI data. Neurosci Biobehav Rev 2017;83:240-51. https://doi.org/10.1016/j.neubiorev.2017.10.017

[33] Aly M, Yonelinas AP, Kishiyama MM, Knight RT. Damage to the lateral prefrontal cortex impairs familiarity but not recollection. Behav Brain Res 2011;225:297-304.

https://doi.org/10.1016/j.bbr.2011.07.043

[34] Perceval G, Martin AK, Copland DA, Laine M, Meinzer M. Multisession transcranial direct current stimulation facilitates verbal learning and memory consolidation in young and older adults. Brain Lang 2020;205:104788. https://doi.org/10.1016/j.bandl.2020.104788

[35] Ghasemian-Shirvan E, Farnad L, Mosayebi-Samani M, Verstraelen S, Meesen RL, Kuo MF, Nitsche MA. Age-related differences of motor cortex plasticity in adults: A transcranial direct current stimulation study. Brain Stimul 2020;13:1588-99. https://doi.org/10.1016/j.brs.2020.09.004

[36] Fertonani A, Brambilla M, Cotelli M, Miniussi C. (2014). The timing of cognitive plasticity in physiological aging: A tDCS study of naming. Front Aging Neurosci 2014;6:131.

https://doi.org/10.3389/fnagi.2014.00131

[37] Polanía R, Nitsche MA, Paulus W. (2011). Modulating functional connectivity patterns and topological functional organization of the human brain with transcranial direct current stimulation. Hum Brain Mapp 2011;32:1236-49. https://doi.org/10.1002/hbm.21104 
[38] Peña-Gómez C, Sala-Lonch R, Junqué C, Clemente IC, Vidal D, Bargalló N, Falcón C, Valls-Solè J, Pascual-Leone A, Bartrés-Faz D. Modulation of large-scale brain networks by transcranial direct current stimulation evidenced by resting-state functional MRI. Brain Stimul 2012;5:252-63. https://doi.org/10.1016/j.brs.2011.08.006

[39] Huo L, Zheng Z, Huang J, Li R, Li J, Li J. Transcranial direct current stimulation enhances episodic memory in healthy older adults by modulating retrieval-specific activation. Neural Plast 2020;8883046. https://doi.org/10.1155/2020/8883046

[40] Andrews SC, Hoy KE, Enticott PG, Daskalakis ZJ, Fitzgerald PB. Improving working memory: The effect of combining cognitive activity and anodal transcranial direct current stimulation to the left dorsolateral prefrontal cortex. Brain Stimul 2011;4:84-9. https://doi.org/10.1016/j.brs.2010.06.004

[41] Deldar Z, Rustamov N, Blanchette I, Piché M. Improving working memory and pain inhibition in older persons using transcranial direct current stimulation. Neurosci Res 2019;148:19-27. https://doi.org/10.1016/j.neures.2018.12.007

[42] Jones KT, Stephens JA, Alam M, Bikson M, Berryhill ME. Longitudinal neurostimulation in older adults improves working memory. PloS one 2015;10:e0121904. https://doi.org/10.1371/journal.pone.0129751

[43] Nissim NR, O'Shea A, Indahlastari A, Kraft JN, Von Mering O, Aksu S, Progres E, Cohen R, Woods AJ. Effects of transcranial direct current stimulation paired with cognitive training on functional connectivity of the working memory network in older adults. Front Aging Neurosci 2019;11:340. https://doi.org/10.3389/fnagi.2019.00340

[44] Stoynova N, Laske C, Plewnia C. Combining electrical stimulation and cognitive control training to reduce concerns about subjective cognitive decline. Brain Stimul 2019;12:1083-85. https://doi.org/10.1016/j.brs.2019.04.008

[45] Mancuso LE, llieva IP, Hamilton RH, Farah MJ. Does transcranial direct current stimulation improve healthy working memory?: a meta-analytic review. J Cogn Neurosci 2016;28:1063-89. https://doi.org/10.1162/jocn_a_00956 